\title{
PERFURAÇÕES ESOFÁGICAS
}

\author{
Esophageal perforations
}

\author{
Maria Aparecida Coelho de Arruda HENRY, Mauro Masson LERCO, Walmar Kerche de OLIVEIRA, \\ Antonio José Maria CATANEO, Daniele Cristina CATANEO, \\ Raul Lopes RUIZ Jr., Rodrigo Severo de Camargo PEREIRA
}

ABCDDV/530

Henry MACA, Lerco MM, Oliveira WK, Cataneo AJM, Cataneo DC, Ruiz Jr RL, Pereira RSC. Perfurações esofágicas. ABCD Arq Bras Cir Dig 2007; 20(2):73-6.

RESUMO - Racional - Dentre as perfurações do trato gastrointestinal, as lesões do esôfago são as de pior prognóstico. Objetivo - Avaliar os aspectos etiológicos, diagnósticos e terapêuticos de pacientes com perfuração esofágica atendidos no Hospital das Clínicas da Faculdade de Medicina de Botucatu - UNESP. Métodos - Avaliação retrospectiva de pacientes internados no período de janeiro de 1999 a dezembro de 2006. Foram estudados 24 pacientes (18 homens e 6 mulheres) com idade média de 52 anos. Os pacientes foram divididos em dois grupos de 12. O Grupo 1 compreendia os pacientes cuja perfuração ocorreu na evolução de câncer do esôfago e o Grupo 2 os pacientes com perfuração devida a causas diversas. No Grupo 2 as causas foram: procedimento endoscópico em três casos, fundoplicatura em três, ingestão de corpo estranho em dois, balão de Blackmore em um, ingestão de antiinflamatório em um, pós-operatório de diverticulectomia em um, ferimento por arma de fogo em um. O esôfago torácico foi o local mais acometido (12 pacientes no Grupo 1 e sete no Grupo 2. Em cinco pacientes do Grupo 1 foi realizada entubação transtumoral e nos demais gastrostomia ou jejunostomia. No Grupo 2, o procedimento realizado nas perfurações do esôfago torácico foi esofagectomia. Resultados - A mortalidade operatória no Grupo 1 foi de $25 \%$ e no Grupo 2 de 8,33\%. Conclusão - a) A lesão do esôfago cervical apresenta, em geral, evolução favorável; b) a conduta cirúrgica, mesmo quando realizado em fase não precoce (primeiras 24 horas), resulta em boa resolução.

DESCRITORES - Esôfago. Perfuração esofágica. Gastrostomia.

\section{INTRODUÇÃO}

A perfuração esofágica constitui importante emergência médica e desafio terapêutico para o cirurgião geral e torácico, devido à gravidade de suas conseqüências. Trata-se de um evento incomum e com quadro clínico inespecífico, fatos que contribuem para o diagnóstico e conseqüente conduta realizados tardiamente ${ }^{16,20}$.

A perfuração esofágica pode ocorrer durante procedimentos endoscópicos ou cirúrgicos ${ }^{13,24}$, trauma ${ }^{6}$, ferimentos por projéteis ou arma branca ${ }^{7,22}$, após ingestão de corpos estranhos $^{3}$, medicamentos ${ }^{10,11}$, substâncias cáusticas ${ }^{12}$, na evolução de tumores do esôfago ${ }^{1}$ e até espontaneamente (síndrome de Boerhaave).

$\mathrm{O}$ estabelecimento de uma conduta normativa para a perfuração esofágica traz dificuldades, pois ela depende de vários fatores, sendo as mais importantes a localização, a causa, a presença de lesões associadas e o tempo decorrido entre a ruptura e o tratamento.

Este trabalho teve por objetivo apresentar os aspectos clínicos de perfurações esofágicas em pacientes atendidos pelos autores.

Trabalho realizado no Departamento de Cirurgia e Ortopedia da Faculdade de Medicina de Botucatu - UNESP, Botucatu, SP, Brasil

Endereço para correspondência: Maria Aparecida Coelho de Arruda Henry, e-mail: rhenry@ibb.unesp.br

\section{MÉTODOS}

Foram avaliados retrospectivamente 24 pacientes ( 18 homens e 6 mulheres), com idades variando entre 24 e 73 anos (média 52 anos), atendidos no período de janeiro de 1999 a dezembro de 2006. Os pacientes foram divididos em dois grupos de 12, na dependência da causa da perfuração esofágica:

Grupo 1: pacientes cuja perfuração ocorreu na evolução de câncer esofágico (idade média: 56,7 anos).

Grupo 2: pacientes com perfuração esofágica devida a causas diversas (idade média: 44,9 anos).

A etiologia das perfurações esofágicas encontra-se na Tabela 1.

TABELA 1 - Etiologia e local da lesão em 24 casos de perfuração esofágica

\begin{tabular}{lccc}
\hline Etiologia & $\mathbf{n}$ & $\mathbf{\%}$ & Local da lesão \\
\hline Câncer do esôfago & 12 & 50 & torácico \\
Procedimento endoscópico & 3 & 12,5 & torácico \\
Procedimento cirúrgico (VAR) & 3 & 12,5 & abdominal \\
Ingestão de corpo estranho & 2 & 8,3 & $\begin{array}{l}\text { cervical/ } \\
\text { torácica }\end{array}$ \\
Balão de Blackmore & 1 & 8,3 & torácico \\
Ingestão de antiinflamatório & 1 & 4,1 & torácico \\
Pós-operatório de diverticulectomia & 1 & 4,1 & cervical \\
Ferimento por arma de fogo & 1 & 4,1 & torácico \\
\hline Total & $\mathbf{2 4}$ & $\mathbf{1 0 0 , 0 0}$ & \\
\hline
\end{tabular}

VAR: válvula antirefluxo gastroesofágico 
A análise dos prontuários permitiu o estudo dos aspectos diagnósticos, terapêuticos e evolutivos dos pacientes.

Nos pacientes do Grupo 1 o diagnóstico de perfuração esofágica foi suspeitado pela queixa de tosse de repetição, que se exacerbava durante a alimentação. A confirmação diagnóstica foi realizada pela realização de esofagograma com bário diluído (Figura 1).

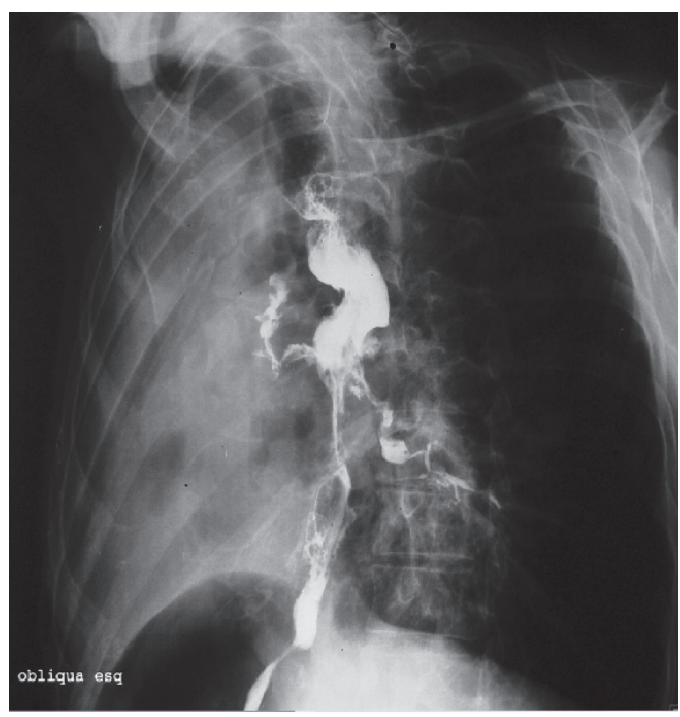

FIGURA 1 - Esofagograma demonstrando fístula esôfagobrônquica

Após antibioticoterapia os pacientes foram submetidos a tratamento cirúrgico paliativo. Cinco foram submetidos à entubação transtumora ${ }^{14} \mathrm{e}$ nos demais foi realizada esofagostomia associada à gastrostomia ou jejunostomia.

Os pacientes do Grupo 2 apresentaram quadro clínico bastante diferenciado, considerando o local da perfuração.

Naqueles com lesão no esôfago cervical, o diagnóstico foi baseado na queixa de dor e abaulamento no pescoço. $\mathrm{O}$ primeiro paciente havia sido submetido à ressecção de divertículo de Zenker há 3 dias e o segundo, deglutido prótese dentária. Nos dois doentes foi realizada sutura da lesão e drenagem.

Dos sete pacientes com lesão no esôfago torácico, foi realizada esofagectomia subtotal, esofagostomia, jejunostomia e decorticação pulmonar em cinco deles. A razão desta conduta foi a positividade do teste de azul de metileno indicando a presença de fístula de alto débito (Figura 2). Nestes, a esofagogastroplastia foi efetuada em segundo tempo. Nos outros dois, foi realizada apenas drenagem pleural.

Nos pacientes com lesão no segmento abdominal do esôfago, foi realizada sutura da lesão, válvula anti-refluxo gastroesofágico total e jejunostomia. Em um deles foi realizada também drenagem do mediastino.

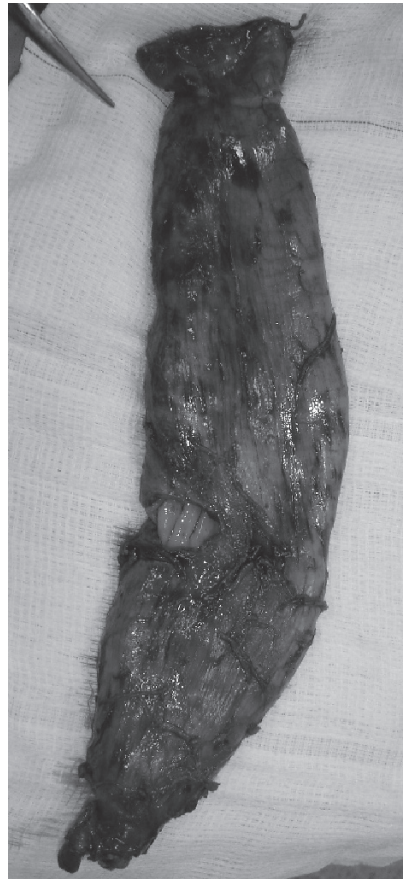

FIGURA 2 - Produto de esofagectomia em paciente com perfuração do esôfago causada por ingestão de antiinflamatório

\section{RESULTADOS}

No Grupo 1, dos cinco pacientes submetidos à entubação transtumoral, um faleceu 14 dias após o procedimento. Evolução desfavorável ocorreu em mais dois pacientes submetidos à esofagostomia associada à jejunostomia, cujos óbitos foram observados nos $7^{\circ}$ e $19^{\circ}$ dias de pósoperatório, respectivamente. Assim, neste grupo a mortalidade foi de $25 \%$.

No Grupo 2, os pacientes com fístulas cervicais apresentaram excelente resposta terapêutica, com remissão precoce dos sintomas.

Os doentes com perfuração torácica e submetidos à esofagectomia subtotal evoluíram bem, sendo a reconstrução do trânsito digestivo realizada em segundo tempo. Nos outros dois pacientes submetidos à drenagem pleural houve remissão total dos sintomas.

O único óbito observado no Grupo 2, ocorreu em paciente submetido à válvula anti-refluxo gastroesofágico videolaparoscópica, onde a perfuração do esôfago abdominal durante a dissecção do mesmo não foi detectada no intra-operatório. O paciente foi submetido à drenagem do mediastino, sutura da lesão, nova fundoplicatura e jejunostomia. Desenvolveu sepse, insuficiência de múltiplos órgãos e óbito no $30^{\circ}$ dia de pós-operatório. Nos outros dois doentes com perfuração do esôfago abdominal, a sutura da lesão e fundoplicatura resultaram em excelente evolução. 


\section{DISCUSSÃO}

Cabe a Barrett, não só a descrição da importante afecção esofágica que leva seu nome ${ }^{4}$, como também o pioneirismo no tratamento cirúrgico da perfuração esofágica. Foi este eminente médico australiano o primeiro a realizar com sucesso o tratamento cirúrgico de um paciente com síndrome de Boerhaarve 5 .

Apesar dos grandes avanços observados na área cirúrgica nas últimas décadas, com novas técnicas, advento da videocirurgia, aparelhos de ventilação mecânica, monitoramento hemodinâmico, nutrição parenteral total e antibióticos, o prognóstico dos pacientes com perfuração esofágica continua sombrio ${ }^{16,18,19,26,27}$.

Nas inúmeras casuísticas apresentadas, observa-se que os autores não fazem referência a perfurações esofágicas detectadas nos portadores de câncer do esôfago. Todavia Pinotti e Ellenbogen ${ }^{20}$ referem em seu artigo de revisão, que as afecções neoplásicas e inflamatórias do esôfago constituem fatores predisponentes de perfurações deste órgão. Assim, julga-se oportuna a inclusão de pacientes com câncer do esôfago nesta casuística.

Os 12 pacientes do Grupo 1 fazem parte de uma série de 120 pacientes com câncer de esôfago atendidos em igual período nesta instituição. Embora não fosse objetivo deste estudo analisar a incidência de perfurações nesse grupo como um todo, é interessante relatar que ela foi de $10 \%$ - superior à publicada por Martini et al. ${ }^{15} \mathrm{e}$ Weaver e Matthews ${ }^{25}$-, e o carcinoma espinocelular foi o tipo histológico observado na maioria dos pacientes (11), havendo apenas um doente com adenocarcinoma.

A fístula esofagobrônquica ocorreu após tratamento radioterápico em dois pacientes e nos demais este tratamento foi contraindicado pela presença da fístula.

A oclusão eficiente da fístula esofagobrônquica nos pacientes com câncer do esôfago é observada em $80 \%$ dos casos submetidos à entubação tumoral ${ }^{14,17}$. Estes procedimentos podem ser realizados com próteses plásticas ou metálicas auto-expansíveis. O risco de complicações com a prótese auto-expansível é menor, porém ela possui o inconveniente de seu alto custo. Nos pacientes desta série foi utilizada a prótese plástica.

A sobrevida dos pacientes do Grupo 1 variou entre 2 e 19 meses, com média de 4 meses, semelhante à relatada por Andreollo et a ${ }^{1}$. Os óbitos foram decorrentes de complicações infecciosas das vias respiratórias.

Nos pacientes do Grupo 2, a região esofágica mais acometida foi a torácica (Tabela 1).

Dos sete pacientes com lesão no esôfago torácico, cinco foram submetidos à esofagectomia subtotal, esofagostomia e jejunostomia. Em apenas um paciente, cuja perfuração foi decorrente de dilatação endoscópica com olivas de Eder-Puestow, a operação foi realizada imediatamente após a lesão. Os outros quatro pacientes foram encaminhados de outros serviços e dois deles com drenagem pleural à direita. Nestes, a ressecção esofágica foi realizada de 36 a 48 horas após a perfuração. Nos cinco pacientes a esofagogastroplastia foi postergada para 3 a 6 meses após o primeiro tempo cirúrgico. Esta conduta foi adotada também por Corsi et $\mathrm{al}^{7}$.

Nos outros dois pacientes com lesão na porção torácica do esôfago, as causas foram ingestão de corpo estranho (prótese) e procedimento endoscópico. A sutura em um plano, seguida de drenagem pleural foi realizada nos dois pacientes com bons resultados. Nestes, não houve necessidade de tamponamento da sutura com retalho pleural, feixe muscular intercostal ou outras estruturas, conforme preconizado por Zwischenberger et $\mathrm{al}^{28}$.

A lesão no esôfago cervical foi observada em dois pacientes (Tabela 1). No primeiro, a perfuração foi causada por ingestão de corpo estranho (prótese) e no segundo, por deiscência de sutura esofágica após diverticulectomia de Zenker. Os dois pacientes foram submetidos à cervicotomia esquerda, sutura da lesão e drenagem, com bons resultados.

A benignidade dos dois pacientes citados contrasta com a gravidade dos casos relatados por Steimann et a ${ }^{22}$. De 34 pacientes com lesão cervical estudados por estes autores, 25 tiveram como etiologia ferimento por arma de fogo, oito por arma branca e um por trauma fechado. Lesões associadas de traquéia, coluna vertebral, vasos, nervo recorrente, mandíbula, hióide e tireóide foram observadas na maioria dos doentes e exigiram conduta cirúrgica nas primeiras 12 horas após a lesão, com boa evolução.

A análise da literatura permite afirmar que os cirurgiões nacionais que atuam em grandes centros urbanos (Tabela 2) são os autores das maiores casuísticas relacionadas ao trauma esofágico provocado por projéteis. Este fato reflete o elevado grau de violência das grandes cidades brasileiras.

TABELA 2 - Perfurações esofágicas: contribuições nacionais ao tema

\begin{tabular}{|c|c|c|c|c|c|}
\hline Autor & Casuística & $\begin{array}{c}\text { Projétil de } \\
\text { arma de } \\
\text { fogo (n) }\end{array}$ & $\%$ & Mortalidade & Localidade \\
\hline Fujimura et al 1977 & 25 & 12 & 48 & $40 \%$ & São Paulo \\
\hline Steiman et al 1990 & 45 & 34 & 75 & $15,5 \%$ & São Paulo \\
\hline Corsi et al 1996 & 944 & 9 & 100 & $44 \%$ & São Paulo \\
\hline $\begin{array}{l}\text { Normando et al } \\
2006\end{array}$ & 41 & 14 & 32 & $37,2 \%$ & Belém \\
\hline Narciso et al 2003 & 30 & 27 & 67 & $22 \%$ & $\begin{array}{l}\text { Rio de } \\
\text { Janeiro }\end{array}$ \\
\hline $\begin{array}{l}\text { Assunção Silva } \\
1987\end{array}$ & 9 & 9 & 30 & $36,6 \%$ & $\begin{array}{l}\text { Rio de } \\
\text { Janeiro }\end{array}$ \\
\hline Thomson et al 1987 & 44 & 5 & 22 & $0 \%$ & Londrina \\
\hline $\begin{array}{l}\text { Ximenes-Neto et } \\
\text { al } 1982\end{array}$ & 21 & 2 & 11 & $36 \%$ & Brasília \\
\hline
\end{tabular}

A lesão do esôfago abdominal foi observada em três pacientes desta série, resultantes de iatrogenia, durante o tratamento cirúrgico da doença do refluxo gastroesofágico. A conduta adotada foi a sutura da lesão e nova fundoplicatura, com resultado satisfatório em dois deles. Todavia, no terceiro paciente esta conduta não foi suficiente, pois ele desenvolveu mediastinite, provavelmente pela drenagem da secreção gástrica através do hiato esofágico. Apesar da realização de toracotomia direita e drenagem do medias- 
tino, o paciente desenvolveu sepse e óbito no $30^{\circ}$ dia de pós-operatório.

Embora a maioria dos autores mencione que, dentre as perfurações esofágicas a de pior prognóstico é aquela localizada na porção torácica, na presente casuística o único óbito ocorreu em paciente com lesão abdominal ${ }^{9,20,21}$. Todavia a afecção responsável pela evolução desfavorável deste paciente foi torácica (grave mediastinite).

O índice de sobrevida dos pacientes desta série (91\%) reflete a superioridade da conduta cirúrgica em relação à conservadora, em conformidade com os relatos de vários autores $2,8,22,23,26$.

\section{CONCLUSÕES}

a) Dos três segmentos esofágicos, o cervical é a sede de lesões com melhor prognóstico, desde que não acompanhado de comprometimento de órgãos vitais próximos a ele; b) nas lesões do esôfago torácico a conduta mais adequada é cirúrgica, mesmo se realizada após a fase considerada precoce de 24 horas.

Henry MACA, Lerco MM, Oliveira WK, Cataneo AJM, Cataneo DC, Ruiz Jr. RL, Pereira RSC. Esophageal perforations. ABCD Arq Bras Cir Dig 2007; 20(2):73-6.

ABSTRACT - Background - Among perforations of the gastrointestinal tract, esophageal lesions are of worst prognosis. Aim - To evaluate the etiology, frequency, diagnosis and therapeutic applied in patients with esophageal perforations assisted at the clinical hospital of the Botucatu School of Medicine - UNESP. Methods - This is a retrospective assessment of patients hospitalized from January 1999 to December 2006. Twenty-four patients (18 males and 6 females) with a mean age of 52 years were studied. Patients were divided into 2 groups of 12 individuals each: Group 1: patients whose perforation occurred during esophageal cancer development, and Group 2: patients with perforation resulting from various causes. In Group 2, the causes were endoscopic procedure (3), fundoplication (3), foreign body ingestion (2), Blackmore balloon (1), anti-inflammatory drug ingestion (1), diverticulectomy postoperative period (1), firearm wound (1). Thoracic esophagus was the most frequently affected site 12 patients in Group 1 and 7 in Group 2. In 5 patients from Group 1, transtumoral intubation was performed, and gastrostomy or jejunostomy was carried out in the others. In Group 2, the procedure adopted for thoracic perforations was esophagectomy. Results - Operative mortality in Group 1 was $25 \%$, and in Group 2 it was of $8.33 \%$. Conclusions - a) Cervical esophageal lesion generally presents favorable development; b) surgical treatment, even when performed at a non-early phase (first 24 hours) results in good evolution.

HEADINGS - Esophagus. Esophageal perforation. Gastrostomy.

\section{REFERÊNCIAS}

1. Andreollo NA, Brandalise NA, Lopes LR, Leonardi LS. Fístula esofagobrônquicas. Acta Oncol Bras. 1987;7:129-34.

2. Assunção-Silva HF, Biasi P, Biasi S, Lima OL, Júdice LF. Perfurações traumáticas do esôfago - experiência de 30 casos. Rev Bras Cir. 1987;77:13-5.

3. Barillo JL, Fontes LE, Koury Filho M, Guimarães RI, Silveira OH. Mediastinite após deglutição de espinha de peixe. Rev Col Bras Cir. 2005;32 (Supl 2):500.

4. Barrett NR. The lower esophagus lined by columnar epithelium. Surgery 1957;41:881-94

5. Barrett NR. Report of a case of spontaneous perforation of the esophagus successfully treated by operation. Br J Surg. 1947;35:216-8.

6. Chen A, Park D, Sihler KC, O'Keefe G. Delayed tracheoesophageal fistula formation after blunt trauma. Chest. 2006;130:308s.

7. Corsi PR, Pinto LE, Pinto MC, Rasslan S. Ferimentos penetrantes do esôfago torácico. Rev Col Bras Cir. 1996;23:73-5.

8. Fujimura I, Lemos PCP, Leite RCC, Altenfelder PF, Warde PJ, Oliveira MR. Lesões perfurativas do esôfago: estudo de 25 casos. Rev Paul Med. 1977;90:5966.

9. Goldenberg S, Oliveira E, Figueira A, Herani Filho B. Perfuração instrumental do esôfago. Rev Assoc Med Bras. 1972;18:103-8.

10. Goldenzon Filho I, Mattos LAGJ, Ehrhardt AO. Lesões esofágicas por medicamentos. J Bras Gastroenterol. 2005;5:21-4.

11. Henry MACA, Lerco M M, Oliveira W K, Dias FG. Perfuração do esôfago causada pela ingestão de anti inflamatórios. Rev Col Bras Cir. 2005;32 (Supl 2):372.

12. Henry MACA, Lerco MM, Oliveira WK, Batalha PKM. Lesões esofágicas causadas pela ingestão de substâncias cáusticas ou corrosivas. ABCD Arq Bras Cir Dig. 2005;18:3-7.

13. Korula J, Pandya K, Yamada S. Perforation of esophagus after endoscopic variceal sclerotherapey. Dig Dis Sci. 1989;34:324-7.

14. Malafaia O. Experiência de 15 anos com o tratamento paliativo do câncer do esôfago através da tunelização esofágica. Rev Col Bras Cir. 1986;13:211-5.

15. Martini N, Goodner JT, D'Angio G, Beattie E. Tracheoesophageal fistula due to cancer. J Thorac Cardiovasc Surg. 1970;59:319-24.

16. Michel L, Grillo HC, Malt RA. Operative and non operative management of esophageal perforations. Ann Surg. 1981;194:57-63.
17. Moura EGH. Câncer avançado do esôfago: tratamento paliativo e das complicações. In: Sterchele R, editor. Complicações em cirurgias do aparelho digestivo. São Paulo: Fungastro; 1999. p.7-24.

18. Narciso GA, Azevedo DE, Guimarães CA, Mathias I, Azevedo LG, Machado T. Perfurações do esôfago. Rev Col Bras Cir. 2003;30:216-23.

19. Normando Jr GR, Tavares MA, Azevedo IV, Modesto A, Janahú AJ, Feliz AH. Mediastinite por perfuração e ruptura do esôfago torácico. Rev Col Bras Cir. 2006;33:361-4.

20. Pinotti HW, Ellenbogen G. Traumatismos do esôfago. J Pneumol. 1979;5:1537 .

21. Popovsky J, Lee YC, Berk JL. Gunsshot wounds of the esophagus. J Thorac Cardiovasc Surg. 1976;72:609-12.

22. Steinman E, Utiyama EM, Pires PWA, Birolini D. Ferimentos traumáticos de esôfago. Rev Hosp Clin Fac Med S Paulo. 1990;43:127-31.

23. Thonson JC, Jeolás LC, Souza JC, Villari Filho S, Freitas MA. Perfuração do esôfago: estudo de nove casos. Rev Col Bras Cir. 1987;14:5-8.

24. Waked A, Murakami HL, Bastos AA. Perfuração do esôfago cervical durante entubação oro traqueal. Rev Col Bras Cir. 2005;32 (Supl 2):580.

25. Weaver RM, Matthews HR. Palliation and survival in malignant oesophagorespiratory fistula .Br J Surg. 1980;67:539-42.

26. Ximenes-Neto M, Silva RO, Fleury Jr I. Perfuração do esôfago. Rev Bras Cir. 1982;72:351-8.

27. Zumbro GL, Anstadt MP, Mawulawde K, Bhimji S, Paliotta MA, Pai G. Surgical management of esophageal perforations: role of esophageal conservation in delayed perforation. Am Surg. 2002;68:36-40.

28. Zwischenberger JB, Alpard SK, Orringer MB. Esôfago. In: Townsens CM, Beuchamp RD, Evers BM, Matox KL, editores. Sabiston. Tratado de cirurgia: as bases biológicas da prática cirúrgica moderna. 16a ed. Rio de Janeiro: Guanabara Koogan; 2002. p.794-8.

Conflito de interesse: não há Fonte financiadora: não há Recebido para publicação em: 13/01/2007 Aceito para publicação em: 07/04/2007 